\title{
ON SOME IMPROPERLY POSED PROBLEMS FOR QUASILINEAR EQUATIONS OF MIXED TYPE( $\left.{ }^{1}\right)$
}

BY

\author{
L. E. PAYNE AND D. SATHER
}

1. Introduction. In a previous paper [6] the authors considered certain improperly posed initial-boundary value problems for the Chaplygin equation

$$
\frac{\partial^{2} u}{\partial y^{2}}+h(y) \frac{\partial^{2} u}{\partial x^{2}}=0
$$

The Chaplygin equation, which arises in the study of transonic flow, is one of the simplest equations of mixed type. It was shown that with appropriate hypotheses on the function $h$ a problem of this type could be stabilized (i.e., the solution made to depend continuously on the data) by restricting the solution to lie in the class of functions whose $L_{2}$ integrals are bounded by some prescribed constant $M$. Similar restrictions have previously been shown (see e.g., [2], [7]) to guarantee continuous dependence in certain improperly posed problems for elliptic and parabolic equations. However, little study has up to now been devoted to improperly posed problems for equations of mixed type. For that matter one is rarely able to determine just what is a well set problem for such an equation.

In this paper we demonstrate that the convexity arguments which led to stability inequalities for the Chaplygin equation [6] actually can be carried over with some modification to yield stability inequalities and error bounds in improperly posed problems for a wide class of quasilinear partial differential equations of mixed type. We shall be concerned here only with the question of obtaining such inequalities. We leave aside the difficult question of existence. The particular equation which we consider is by no means the most general one amenable to the convexity method. We restrict our attention to a relatively simple equation but one which exhibits most of the troublesome features which would appear in the more general operators.

A survey of the literature on improperly posed problems will appear in a forthcoming paper by Payne [5].

In this paper we consider the following initial-boundary value problem for a cylindrical domain $\Omega=D \times(0, Y)$, where $D$ is an arbitrary $n$-dimensional domain with smooth boundary $\dot{D}$ :

Received by the editors July 1, 1966.

(1) This research was supported in part by the National Science Foundation Grant No. GP 5882 with Cornell University. 
Problem A.

$$
\begin{aligned}
L u= & u_{, y y}-\left(a_{i j} u_{, i}\right)_{, j}-f\left(x, y, u, u_{, y}\right)=0 \text { in } \Omega, \\
& u \text { prescribed on } \dot{\Omega}=\dot{D} \times[0, Y], \\
& u, \frac{\partial u}{\partial y} \text { prescribed on } y=0 .
\end{aligned}
$$

Here $\left(a_{i j}\right)$ is a symmetric matrix function of the variables $x=\left(x_{1}, x_{2}, \ldots, x_{n}\right)$ and $y$. The matrix $\left(a_{i j}\right)$ is not assumed to be definite or even semidefinite. The repeated index is used to designate summation from 1 to $n$ and $u_{, i}=\partial u / \partial x_{i}$. The function $f$ is to be Lipschitz continuous in its last two arguments, i.e.,

$$
\left|f\left(x, y, u_{1}, u_{1, y}\right)-f\left(x, y, u_{2}, u_{2, y}\right)\right| \leqq M_{1}\left|u_{1}-u_{2}\right|+M_{2}\left|u_{1, y}-u_{2, y}\right| .
$$

We assume that there exists a nonnegative constant $\delta$ such that the matrix $\left(B_{i j}\right)$ with elements

$$
B_{i j}=\frac{\partial}{\partial y} a_{i j}-2\left(M_{2}+\delta\right) a_{i j}
$$

is negative semidefinite in $\Omega$. Here $M_{2}$ is given by (1.4).

We shall say that $u$ belongs to $\mathscr{M}$ if the condition

$$
\int_{\Omega} u^{2} d x d y \leqq M^{2}
$$

is satisfied for some prescribed constant $M$. In the following section we will develop a priori inequalities which for solutions $u$ of Problem A that belong to $\mathscr{M}$ may be used to obtain uniqueness, continuous dependence on the data and $L_{2}$ bounds on compact subdomains of $\Omega$.

2. The a priori inequalities. We derive the basic inequalities in a form which yields immediately the desired $L_{2}$ bounds; by specialization, these inequalities imply also the properties of uniqueness and continuous dependence on the data.

Let us approximate $u$ by a function $\varphi$ which is piecewise $C^{2}$ in $\Omega, C^{1}$ in $\bar{\Omega}$ and such that $u=\varphi$ on $\dot{\Omega}$. We set $\Omega_{y}=D \times(0, y), 0<y \leqq Y$, and

$$
v=u-\varphi \text {. }
$$

Let $\Omega_{0}$ denote the intersection of $\bar{\Omega}$ and the initial plane $y=0$ and let $\mathscr{F}$ denote the functional

$$
\mathscr{F}(v, y)=\log F(v, y)+c \sigma^{2}, \quad 0<y<Y
$$

where

$$
\sigma=\left(y_{0}+y\right)^{-\alpha}, \quad \alpha>0,
$$

and

$$
\begin{aligned}
F(v, y)=\int_{\Omega_{y}} v^{2} d x d \eta & +(Y-y) \int_{\Omega_{0}} v^{2} d x+k_{1} \int_{\Omega_{0}} v^{2} d x \\
& +k_{2} \int_{\Omega_{0}}\left(v_{, i} v_{, i}+v_{, y}^{2}\right) d x+k_{3} \int_{\Omega}[L \varphi]^{2} d x d y \\
\equiv \int_{\Omega_{y}} v^{2} d x d \eta+Q^{2} &
\end{aligned}
$$


Here the constants $\alpha, c$, and $k_{i}$ in (2.2) through (2.4) are to be determined. The positive constant $y_{0}$ is introduced so that $\sigma$ ranges over a finite interval. We note that $Q$ in (2.4) involves only data terms of $u-\varphi$ and, hence, for fixed $k_{i}$ it will be small if the data of $\varphi$ approximate the data of $u$ sufficiently well.

Our a priori inequalities are a consequence of the following convexity argument. If we can show that the functional $\mathscr{F}$ is a convex function of $\sigma$ then an application of Jensen's inequality yields

$$
\begin{aligned}
e^{c \sigma^{2}}\left[\int_{\Omega_{y}} v^{2} d x\right. & \left.d \eta+Q^{2}\right] \\
& \leqq\left\{e^{c \Sigma^{2}}\left[\int_{\Omega} v^{2} d x d y+Q^{2}\right]\right\}^{\left(\sigma_{0}-\sigma\right) /\left(\sigma_{0}-\Sigma\right)}\left\{e^{c \sigma_{0}^{2}} Q^{2}\right\}^{(\sigma-\Sigma) /\left(\sigma_{0}-\Sigma\right)}
\end{aligned}
$$

where

$$
\Sigma=\left(Y+y_{0}\right)^{-\alpha} \text { and } \sigma_{0}=y_{0}^{-\alpha} \text {. }
$$

Since $u$ is to satisfy (1.6) and $\varphi$ is at our disposal we may assume without loss of generality that

$$
\int_{\Omega} v^{2} d x d y \leqq M_{0}^{2}
$$

In addition, since $Q$ involves only data terms we have

$$
Q^{2} \leqq \nu M_{0}^{2}
$$

where $\nu$ is a computable constant. Therefore

$$
\int_{\Omega_{y}} v^{2} d x d \eta+Q^{2} \leqq e^{c\left(\sigma_{0}-\sigma\right)(\sigma-\Sigma)}\left\{(1+\nu) M_{0}^{2}\right\}^{\left(\sigma_{0}-\sigma\right) /\left(\sigma_{0}-\Sigma\right)} Q^{2(\sigma-\Sigma) /\left(\sigma_{0}-\Sigma\right)} .
$$

This last inequality yields the desired $L_{2}$ bounds for $u$ on compact subdomains of $\Omega$.

Let us now show that $\mathscr{F}$ is a convex function of $\sigma$. We note that

$$
F^{2} \mathscr{F}^{\prime \prime}=F F^{\prime \prime}-\left(F^{\prime}\right)^{2}+2 c F^{2},
$$

where the prime denotes differentiation with respect to $\sigma$. Therefore, in order to establish the convexity of $\mathscr{F}$, it is sufficient to show that the right-hand side of (2.10) is positive. Since

$$
\begin{gathered}
F^{\prime}=-\alpha^{-1} \sigma^{-(1+\alpha) / \alpha} \frac{d F}{d y} \\
F^{\prime \prime}=\alpha^{-2}(1+\alpha) \sigma^{-(1+2 \alpha) / \alpha} \frac{d F}{d y}+\alpha^{-2} \sigma^{-2(1+\alpha) / \alpha} \frac{d^{2} F}{d y^{2}}
\end{gathered}
$$

we have$$
-\alpha \sigma^{(1+\alpha) / \alpha} F^{\prime}=2 \int_{\Omega_{y}} v(x, \eta) v_{, y}(x, \eta) d x d \eta
$$$$
\alpha^{2} \sigma^{2(1+\alpha) / \alpha} F^{\prime \prime}=2(1+\alpha) \sigma^{1 / \alpha} \int_{\Omega_{y}} v v_{, y} d x d \eta
$$$$
+2 \int_{\Omega_{y}} v_{, y}^{2} d x d \eta+2 \int_{\Omega_{0}} v v_{, y} d x+2 \int_{\Omega_{y}} v v_{, y y} d x d \eta
$$ 
The last term in (2.14) may be rewritten as

$$
\begin{aligned}
\int_{\Omega_{y}} v v_{, y y} d x d \eta & =\int_{\Omega_{y}} v\left\{\left(a_{i j} v_{, i}\right)_{, j}-L \varphi+f(u)-f(\varphi)\right\} d x d \eta \\
& =\int_{\Omega_{y}}\left\{-a_{i j} v_{, i} v_{, j}-v L \varphi+v[f(u)-f(\varphi)]\right\} d x d \eta
\end{aligned}
$$

and, hence,

$$
\begin{aligned}
\alpha^{2} \sigma^{2(1+\alpha) / \alpha} F^{\prime \prime}=2(1+\alpha) \sigma^{1 / \alpha} & \int_{\Omega_{y}} v v_{, y} d x d \eta+4 \int_{\Omega_{y}} v_{, y}^{2} d x d \eta \\
& +2 \int_{\Omega_{y}}\{v[f(u)-f(\varphi)]-v L \varphi\} d x d \eta+2 \int_{\Omega_{0}} v v_{, y} d x \\
& -2 \int_{\Omega_{y}}\left(v_{, y}^{2}+a_{i j} v_{, i} v_{, j}\right) d x d \eta .
\end{aligned}
$$

An upper bound for the last term in (2.16) is obtained as follows. We note that for $0<t<y$

$$
\begin{aligned}
0=2 \int_{\Omega_{t}}(t-\eta) v_{, y}\left\{v_{, y y}-\right. & \left.\left(a_{i j} v_{, i}\right)_{, j}+L \varphi-[f(u)-f(\varphi)]\right\} d x d \eta \\
=2 \int_{\Omega_{t}}(t-\eta) v_{, y}\{L \varphi- & {[f(u)-f(\varphi)]\} d x d \eta } \\
& +\int_{\Omega_{t}}\left(v_{, y}^{2}+a_{i j} v_{, i} v_{, j}\right) d x d \eta-t \int_{\Omega_{0}}\left(v_{, y}^{2}+a_{i j} v_{, i} v_{, j}\right) d x \\
& -\int_{\Omega_{t}}(t-\eta) a_{i j, \eta} v_{, i} v_{, j} d x d \eta .
\end{aligned}
$$

Let us now assume that the constant $\delta$ in (1.5) is positive. An application of the arithmetic-geometric mean inequality, and (1.4), (1.5), and (2.17) imply

$$
\frac{d J(t)}{d t} \leqq I(y)+2\left(M_{2}+\delta\right) J(t)
$$

where

$$
J(t)=\int_{\Omega_{t}}(t-\eta)\left(v_{, y}^{2}+a_{i j} v_{, i} v_{, j}\right) d x d \eta
$$

$$
I(y)=y \int_{\Omega_{0}}\left(v_{, y}^{2}+a_{i j} v_{, i} v_{, j}\right) d x+2 M_{1} y \int_{\Omega_{y}}\left|v v_{, y}\right| d x d \eta+\frac{Y}{4 \delta} \int_{\Omega}[L \varphi]^{2} d x d \eta
$$

Integrating (2.18) from 0 to $y$ we obtain

$$
\int_{\Omega_{y}}\left(v_{, y}^{2}+a_{i j} v_{, i} v_{, j}\right) d x d \eta \leqq I(y) e^{2\left(M_{2}+\delta\right) y} .
$$


Substituting (2.21) into (2.16) and using (1.4) we have for some computable constants $b_{i}$ that

$$
\begin{aligned}
& \alpha^{2} \sigma^{2(1+\alpha) / \alpha}\left[F F^{\prime \prime}-\left(F^{\prime}\right)^{2}\right] \\
& \geqq 2(1+\alpha) F \sigma^{1 / \alpha} \int_{\Omega_{y}} v v_{, y} d x d \eta+4 S^{2}+4 Q^{2} \int_{\Omega_{y}} v_{, y}^{2} d x d \eta \\
& \quad-F\left\{b_{1} \int_{\Omega_{0}} v^{2} d x+b_{2} \int_{\Omega_{0}} v_{, y}^{2} d x+b_{3} \int_{\Omega_{0}} v_{, i} v_{, i} d x\right. \\
& \left.\quad+b_{4} \int_{\Omega}[L \varphi]^{2} d x d y+b_{5} \int_{\Omega_{y}} v^{2} d x d \eta+4 b_{6} \int_{\Omega_{y}}\left|v v_{, y}\right| d x d \eta\right\}
\end{aligned}
$$

where

$$
S^{2}=\left(\int_{\Omega_{y}} v^{2} d x d \eta\right)\left(\int_{\Omega_{y}} v_{, y}^{2} d x d \eta\right)-\left(\int_{\Omega_{y}} v v_{, y} d x d \eta\right)^{2}
$$

Since

$$
\int_{\Omega_{y}} v v_{, y} d x d \eta \geqq\left|\int_{\Omega_{y}} v v_{, y} d x d \eta\right|-\int_{\Omega_{0}} v^{2} d x
$$

and

$$
\int_{\Omega_{y}}\left|v v_{, y}\right| d x d \eta \leqq S+\left|\int_{\Omega_{y}} v v_{, y} d x d \eta\right|
$$

it follows from (2.22) that

$$
\begin{aligned}
& \alpha^{2} \sigma^{2(1+\alpha) / \alpha}\left[F F^{\prime \prime}-\left(F^{\prime}\right)^{2}\right] \\
& \geqq 2 F\left[(1+\alpha) \sigma^{1 / \alpha}-2 b_{6}\right]\left|\int_{\Omega_{y}} v v_{, y} d x d \eta\right|+\left(2 S-b_{6} F\right)^{2}-b_{6}^{2} F^{2} \\
& \quad-F\left\{\left[b_{1}+2(1+\alpha) \sigma^{1 / \alpha}\right] \int_{\Omega_{0}} v^{2} d x+b_{2} \int_{\Omega_{0}} v_{, y}^{2} d x\right. \\
& \left.+b_{3} \int_{\Omega_{0}} v_{, i} v_{, i} d x+b_{4} \int_{\Omega}[L \varphi]^{2} d x d y+b_{5} \int_{\Omega_{y}} v^{2} d x d \eta\right\}
\end{aligned}
$$

If we now choose $1+\alpha=2 b_{6}\left(Y+y_{0}\right)$ then the coefficient of the first term in (2.26) is nonnegative and, hence,

$$
\begin{aligned}
\alpha^{2} \sigma^{2(1+\alpha) / \alpha} F^{2} \mathscr{F}^{\prime \prime} & \geqq F^{2}\left[2 c \alpha^{2}\left(Y+y_{0}\right)^{-2(1+\alpha)}-b_{6}^{2}\right] \\
-F\left\{\left[b_{1}+2(1+\alpha) / y_{0}\right] \int_{\Omega_{0}} v^{2} d x+b_{2} \int_{\Omega_{0}} v_{, y}^{2} d x\right. & \\
& \left.+b_{3} \int_{\Omega_{0}} v_{, i} v_{, i} d x+b_{4} \int_{\Omega}[L \varphi]^{2} d x d y+b_{5} \int_{\Omega_{y}} v^{2} d x d \eta\right\}
\end{aligned}
$$

Clearly the constants $k_{1}, k_{2}, k_{3}$ in $Q$, and $c$ can be chosen so that the right-hand side of (2.27) is positive. In fact for any positive $k_{1}, k_{2}$, and $k_{3}$ we need only take 
$c$ large enough so that the first term in (2.27) is the dominant one. Thus it is possible to choose the constants $\alpha, k_{i}$, and $c$ so that $\mathscr{F}$ is a convex function of $\sigma$. We have established the following result.

THEOREM 1. Suppose that $\delta>0$ in (1.5) and the matrix $\left(B_{i j}\right)$ is negative semidefinite. Let $u$ satisfy (1.2), (1.3), and (1.6), and let $v, \sigma, Q, \Sigma, \sigma_{0}$, and $M_{0}$ be defined as in (2.1), (2.3), (2.4), (2.6), and (2.7), respectively. Then there exists a computable constant $K$ such that

$$
\int_{\Omega_{y}} v^{2} d x d \eta \leqq K M_{0}^{2\left(\sigma_{0}-\sigma\right) /\left(\sigma_{0}-\Sigma\right)} Q^{2(\sigma-\Sigma) /\left(\sigma_{0}-\Sigma\right)}, \quad 0<y<Y .
$$

An immediate consequence of the above discussion is

THEOREM 2. Suppose that the constant $\delta$ in (1.5) is nonnegative. If the matrix $\left(B_{i j}\right)$ is negative semidefinite then any solution of Problem A that is in the class $\mathscr{M}$ of functions whose $L_{2}$ integrals over $\Omega$ are bounded by some constant $M$ will depend continuously in $L_{2}$ on the Cauchy data.

Let us remark that if, in particular, the matrix $\left(a_{i j}\right)$ is independent of $y, \delta=0$ and the function $f$ is independent of $u_{, y}$ then $\left(B_{i j}\right)=0$. Moreover, the requirement on the matrix $\left(B_{i j}\right)$ is a "best possible" condition in the sense that one can give examples where Theorem 2 does not hold when this condition fails to be satisfied [6]. Finally, the restriction $\delta>0$ in Theorem 1 can be replaced by $\delta \geqq 0$ if one imposes additional smoothness requirements on $\varphi$ (see [5], [6]).

As an interesting specific application of these results we let $\Omega$ be the rectangular parallelepiped $\{0<x<X, 0<y<Y, 0<t<T\}$ and consider the following Cauchy problem for the wave equation:

Problem A .

$$
\begin{aligned}
& u_{, y y}=u_{, t t}-u_{, x x} \text { in } \Omega, \\
& u \text { prescribed on } x=0, x=X, t=0, \text { and } t=T, \\
& u, \frac{\partial u}{\partial y} \text { prescribed on } y=0 .
\end{aligned}
$$

Then any solution $u$ of Problem $\mathrm{A}^{\prime}$ that is in the class $\mathscr{M}$ will depend continuously in $L_{2}$ on the Cauchy data (compare also [1], [3], and [4]).

\section{BIBLIOGRAPHY}

1. J. R. Cannon, A Cauchy problem for the wave equation for a space-like region, Le Matematiche 16 (1961), 43-50.

2. F. John, A note on "improper" problems in partial differential equations, Comm. Pure Appl. Math. 8 (1955), 591-594.

3. - Continuous dependence on the data for solutions of partial differential equations with a prescribed bound, Comm. Pure Appl. Math. 13 (1960), 551-585.

4. L. Nirenberg, Uniqueness in Cauchy problems for differential equations with constant leading coefficients, Comm. Pure Appl. Math. 10 (1957), 89-105. 
5. L. Payne, On some non-wellposed problems for partial differential equations, Proc. Sympos. Numerical Solutions of Nonlinear Differential Equations, pp. 239-263, Math. Res. Center, U.S. Army, Univ. of Wisconsin, Madison, Wiley, New York, 1966.

6. L. Payne and D. Sather, On some improperly posed problems for the Chaplygin equation, J. Math. Anal. Appl. (to appear).

7. C. Pucci, Sui problemi di Cauchy non "ben posti," Rend. Accad. Naz. Lincei 18 (1955), 473-477.

\section{CORNELL UNIVERSITY,}

ITHACA, NEW YORK 